\title{
Fundamentals and Experimental Results of Shape Memory Alloys
}

\author{
A.C. Kneissl, K. Mehrabi, M. Bruncko \\ Department of Physical Metallurgy and Materials Testing, University of Leoben, Austria
}

First in this presentation the specific phenomena of shape memory alloys (SMAs), namely one-way effect, two-way effect and pseudoelasticity, will be discussed. Shape memory alloys have been successfully introduced into a variety of technical areas over the past few years. A very promising field for a high-volume application of SMAs in the near future is the actuator technology, since with a shape memory element, a pre-determined response can be obtained very easily by thermal or electric stimulus. From the several modes of using the shape memory effect, the intrinsic two-way shape memory effect (TWSME) is the most suitable to apply in actuators since no resetting force has to be considered in design. However, when dealing with the inherently weak intrinsic two-way shape memory effect, it is essential to elaborate the fatigue process thoroughly. For technical applications it is important to know how changes within the substructure quantitatively and qualitatively affect the magnitude of the effect and possibly the element's dimensions and actuating temperatures.

Shape memory wires were trained under constant stress in order to introduce a uniaxial shape memory effect by a thermomechanical treatment. These investigations were carried out on three different alloy systems (NiTi, NiTiW and CuAlNi) with different microstructures (coldworked and annealed condition, with and without particles, large and small grain size). Several thousand thermal cycles were performed on the trained shape memory elements, continuously observing the changes in the deformation behaviour. The influence of the microstructure on development and stability of the intrinsic two-way shape memory effect will be discussed. Furthermore, this presentation deals with the production of thin specimens of shape memory alloys by melt-spinning and splat-cooling (Fig. 1), the training procedure and their characterization with respect to microstructure and functional properties (Fig. 2).

Key words: Shape memory alloys; NiTi(W); CuAlNi; two-way effect, melt-spinning. 

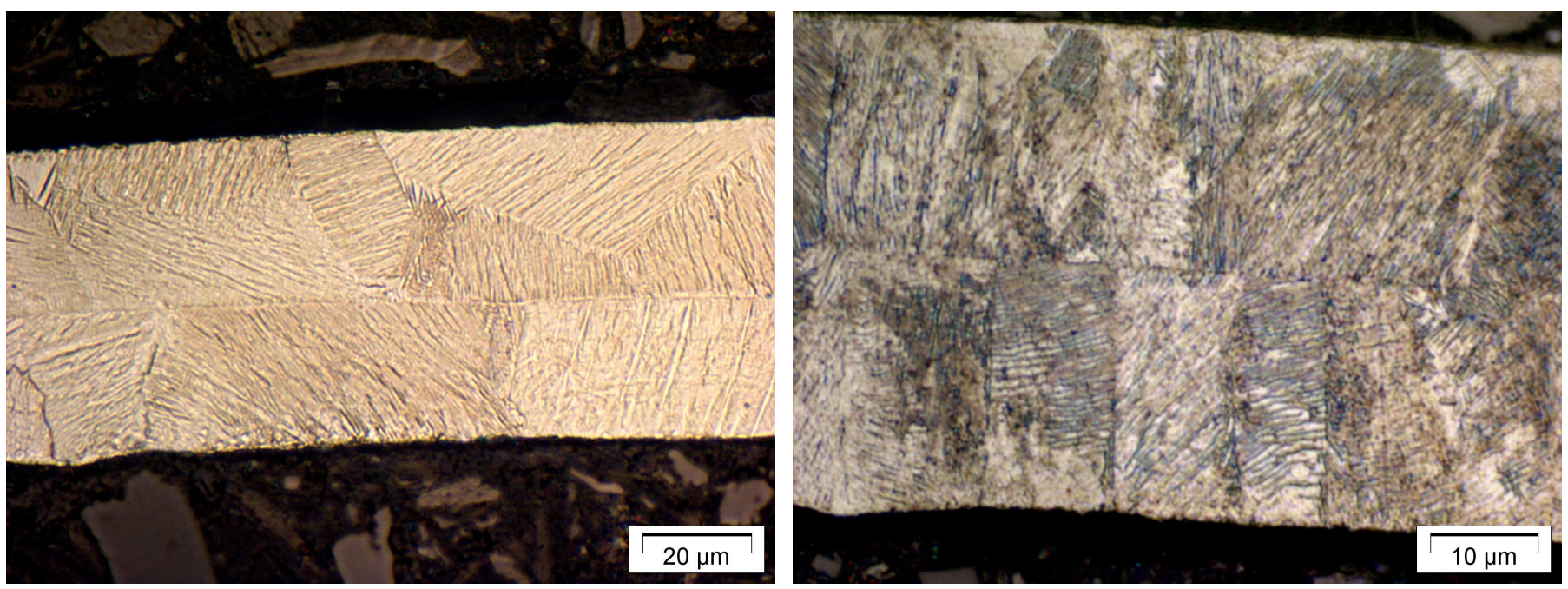

FIG. 1. Martensitic structure of splat-cooled samples.
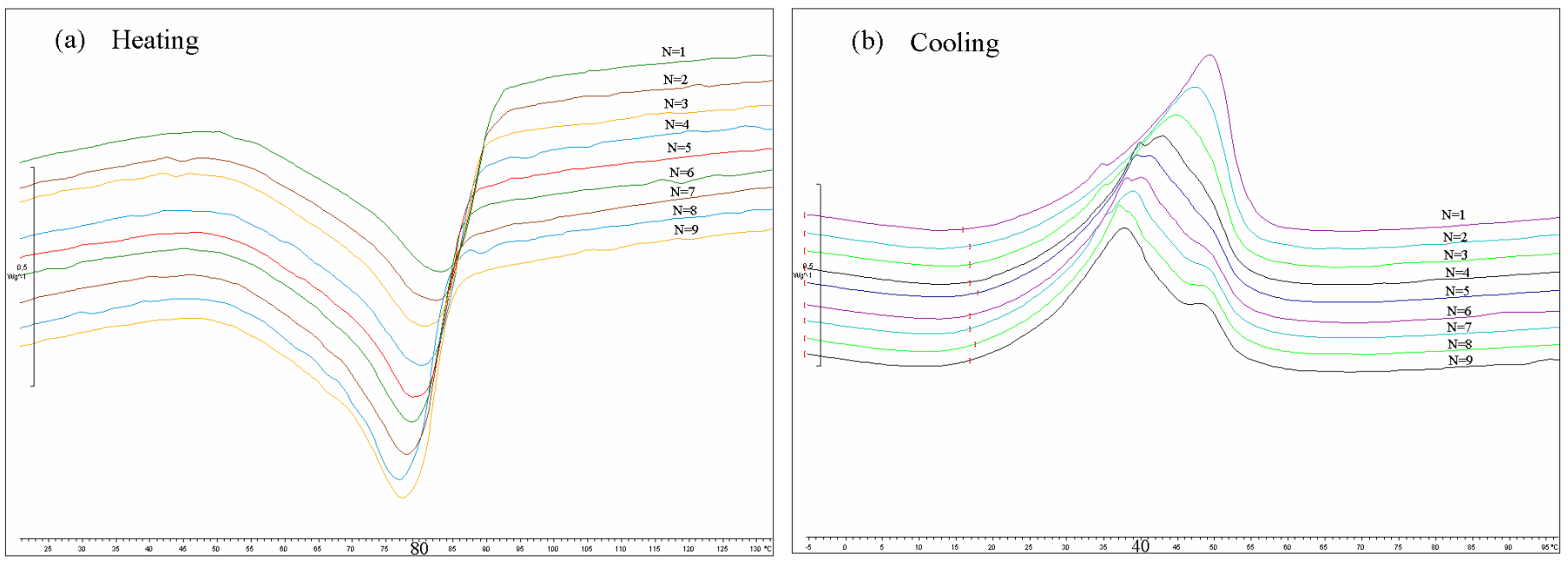

FIG. 2. DSC results of NiTi ribbons after various training cycles 